\title{
Evaluation of the DPC-based inclusive payment system in Japan for cataract operations
}

\author{
$\underline{\text { Nawata, K. }}{ }^{1}$ and K. Kawabuchi ${ }^{2}$ \\ ${ }^{1}$ Graduate School of Engineering, University of Tokyo, Bunkyo-ku, Tokyo 113-8656, Japan \\ ${ }^{2}$ Section of Health Care Economics, Tokyo Medical and Dental University, Bunkyo-ku, Tokyo, 113-8510, \\ Japan
}

Email: nawata@.tmi.t.u-tokyo.ac.jp

\begin{abstract}
Since medical care expenses have been increasing rapidly with the ageing of the population, reducing the length of hospital stay (LOS) has become an important political issue in Japan. A new inclusive payment system based on the diagnosis procedure combination (DPC) was introduced in 82 special functioning hospitals in April 2003. Since April 2004, use of the DPC system has been gradually extended to general hospitals. As of July 2009, a total of 1,283 hospitals, about $14 \%$ of the 8,862 general hospitals in Japan, had joined the DPC system. These 1,283 hospitals have 434,231 beds, which is nearly half of the total beds (913,234 beds) of general hospitals in Japan. The DPC system is an original system developed in Japan. Inclusive payments based on the DPC system cover fees for the following categories only: basic hospital stays, medical checkups, image diagnosis, medication, injections, treatments under 1,000 points (10 yen per point has been paid to hospitals), and medicines used during rehabilitation treatments and related activities. Fees for all other categories, such as fees for operations, are paid on the basis of the conventional fee-forservice system.
\end{abstract}

Unlike the diagnosis-related group/prospective payment system (DRG/PPS) used in the U.S. and other countries, the Japanese DPC system is a per diem prospective payment system. The per diem payment becomes less as the LOS becomes longer. Three periods, Period I, Period II, and Specific Hospitalization Period, are determined for each DPC code. For stays over the Specific Hospitalization Period, the per diem payment is determined through the conventional fee-for-service system. The introduction of the DPC system was one of the largest and most important revisions of the payment system since the Second World War. For the effective use of medical resources, improvement of the DPC system by thorough analyses of the system is absolutely necessary.

In this paper, we first propose a new model that considers heterogeneity of variances. We then present our analysis of the LOS for cataract operations before and after the introduction of the DPC system using the proposed model. The number of cataract patients in Japan has been increasing rapidly with the ageing of the population. According to a survey conducted by the Ministry of Health, Labour and Welfare (2008), nearly 800,000 cataract operations are performed annually and nearly 2.5 billion yen are spent for cataract operations annually. We analyzed the influence of the DPC system and factors that might affect the LOS for cataract patients by examining data collected from 5 general hospitals before and after the introduction of the system. To eliminate the influences of types of operations and treatments, we used data strictly pertaining to the patients who underwent cataract operations and insertion of a prosthetic lens on one eye only. The number of patients was 2,533.

The estimates of the Female, Age 50, Age 90, Not Home dummies are significant and affect the LOS. We found large differences in the changes of average lengths of stay (ALOSs) among hospitals. In hospitals where the ALOSs were long, the ALOSs decreased significantly under the DPC system. On the other hand, in hospitals where the ALOSs were already short, the ALOSs did not decrease under the DPC system. The results of empirical study imply that the DPC system gave strong incentives to reduce the ALOSs for the former hospitals but it gave weak (or no) incentives for the latter hospitals, where the ALOSs were already short.

Keywords: survival analysis, proportional hazard model, length of hospital stay, diagnosis procedure combination (DPC), cataract operation 


\section{INTRODUCTION}

Since medical care expenses have been increasing rapidly with the ageing of the population, reducing the length of hospital stay (LOS) has become an important political issue in Japan. Based on the recommendations of a report submitted by the Central Social Insurance Medical Council concerning the 2002 revision of the Medical Service Fee Schedule, a new inclusive payment system based on the diagnosis procedure combination (DPC) was introduced in 82 special functioning hospitals (i.e., university hospitals, the National Cancer Center, and the National Cardiovascular Center) in April 2003 (Okamura et al., 2005, and Yasunaga et al., 2005). (In the rest of this paper, we refer to the new payment system base on the DPC as the DPC system and to hospitals participating in the system as DPC hospitals.) Since April 2004, the DPC system has been gradually extended to general hospitals. As of July 2009, a total of 1,283 hospitals, about $14 \%$ of the 8,862 general hospitals in Japan, had joined the DPC system. These 1,283 hospitals have 434,231 beds, which is nearly half of the total beds $(913,234$ beds) of general hospitals in Japan. The DPC system is an original system developed in Japan. The DPC classifies diseases, operations, treatments, and patient conditions using a 14-digit code. The first 6 digits classify principal diseases on the basis of the International Classification of Diseases-10 (ICD-10). The remaining 8 digits pertain to information on operations, treatments, and patient conditions such as the presence of a secondary disease. Initially, the DPC system classified patients into 1,860 payment categories. Currently, the number of categories is 1,572. Inclusive payments based on the DPC system cover fees for the following categories only: basic hospital stays, medical checkups, image diagnosis, medication, injections, treatments under 1,000 points (10 yen per point has been paid to hospitals), and medicines used during rehabilitation treatments and related activities. Fees for all other categories, such as fees for operations, are paid on the basis of the conventional fee-for-service system.

Unlike the diagnosis-related group/prospective payment system (DRG/PPS) used in the U.S and other countries, the Japanese DPC system is a per diem prospective payment system. The per diem payment becomes less as the LOS becomes longer. Three periods, Period I, Period II, and Specific Hospitalization Period, are determined for each DPC code. Period I is set as the 25th percentile of the LOS of the surveyed hospitals. Period II is set as the average length of hospital stay, that is, the 50th percentile (although this value is actually the median, it is called the "average length of hospital stay" in the DPC system). Finally, the Specific Hospitalization Period is given by the following equation: (average length of hospital stay) $+2 \times$ (standard deviation). The basic per diem payment is determined according to the length of hospital stay. For stays below Period I, the per diem payment to hospitals is $15 \%$ more than the average per diem payment of the patients whose stays were within the average LOS. For hospital stays between Periods I and II, the per diem payment is determined such that (per diem payment in Period I - average per diem payments) $X$ (number of days in Period I ) equals (the average per diem payments - per diem payment between Periods I and II) $\times$ (number of days between Periods I and II). For stays between Period II and the Specific Hospitalization Period, the per diem payment is reduced by an additional $15 \%$. In the case of cataract operations (DPC code: $021103 \times 01 \times 000$ ), the per diem inclusive payment in 2005 was 2,546 points up to the third day of hospitalization, 1,882 points for the 4th-6th days, and 1,600 points for the 7th-10th days. For stays over the Specific Hospitalization Period, the per diem payment is determined through the conventional fee-for-service system.

The introduction of the DPC system was one of the largest and most important revisions of the payment system since the Second World War. For the effective use of medical resources, improvement of the DPC system by thorough analyses of the system is absolutely necessary. However, thorough evaluations using econometrical models, where individual patient characteristics and treatment types are taken into account, have yet to be performed.

In this paper, we first propose a new model that considers heterogeneity of variances. The model can be easily estimated by a standard statistical package program. We then present our analysis of the LOS for cataract operations before and after the introduction of the DPC system using the proposed model. The number of cataract patients in Japan has been increasing rapidly with the ageing of the population. According to a survey conducted by the Ministry of Health, Labour and Welfare (2008), nearly 800,000 cataract operations are performed annually and nearly 2.5 billion yen are spent for cataract operations annually. We analyzed the influence of the DPC system and factors that might affect the LOS for cataract patients by examining data collected from 5 general hospitals before and after the introduction of the system. To eliminate the influences of types of operations and treatments, we used data strictly pertaining to the patients who underwent cataract operations and insertion of a prosthetic lens on one eye only. The number of patients was 2,533 . 


\section{DATA}

\subsection{Hospitals}

In this study, we use two data sets collected before and after the introduction of the DPC system. The first period was from April 2000 to March 2001, before the introduction of the DPC system. The data set was collected from 36 hospitals participating in the Project for Information Standardization and System Developments for Efficient Hospital Management. For each patient, the dates of admission and discharge from the hospital, date of birth, sex, placement after hospitalization, names of principle and secondary diseases, and types of medical operations and treatments were reported. The names of principle and secondary diseases were based on the International Classification of Diseases 9 (ICD-9) or ICD-10, and the type of operation and treatment was based on the International Classification of Diseases, 9th Revision, Clinical Modification (ICD-9-CM). The second period was from April 2005 to December 2007, after the introduction of the DPC system. The data set was collected from 86 hospitals by the Division of Health Care Economics, Tokyo Medical and Dental University. For each patient, the DPC code, dates of hospitalization and discharge from the hospital, date of birth, sex, placement after hospitalization, ICD-10 code for the principle disease, purpose of hospitalization, presence of secondary disease and the attending treatment if any, and medical payment amounts (including DPC-based, fee-for-service, and total payments) were reported.

When a patient has a secondary disease or complication, the LOS is expected to be longer. To remove these effects, we analyze only patients who underwent one-eye cataract operations and insertion of a prosthetic lens (pseudophakos). Patients who underwent other types of operations or treatments were eliminated from the data set. Owing to the standardization of cataract operations (Fedorowize, Lawrence, and Gettie, 2006), the homogeneity of data in the two sample periods is considered to be high. We analyze the data collected from 5 general hospitals (Hp1-Hp5) where more than 10 patients were reported in both periods. These hospitals have 440 1,109 beds, with an average of 688 beds. One of the hospitals is in Tohoku, one is in Kanto, and three are in Tokai. The managerial organizations include one mutual aid association, two Red Cross and two medical associations, corporations, and other organizations. Two hospitals joined the DPC system in 2004, and three hospitals joined in 2006. The total number of patients included in the analysis is 2,533, including 1,567 for the first period and 966 for the second period.

\subsection{Lengths of Hospital Stay}

In the first period, the average length of stay (ALOS) and standard deviation for all patients were 5.99 days and 10.45 days, respectively. The ALOSs by hospitals were 3.96, 9.28, 8.91, 5.83, and 8.96 days for Hp1$\mathrm{Hp5}$. In the second period, the average and standard deviation for all patients were 4.11 days and 1.08 days, respectively. The ALOS was shortened by 1.48 days, and the standard deviation became $1 / 8$ of that in the first period. The ALOSs by hospital were 4.00, 5.50, 4.18, 3.61, and 6.03 days for Hp1-Hp5, respectively. For four hospitals (Hp2-Hp5), the ALOSs were shortened by $2.22 \sim 4.73$ days. Although the value is small, the ALOS for Hp1, where the ALOS was shortest in the first period, increased by 0.04 day. As a result, the ALOS for Hp4 became the shortest of the hospitals studied.

\section{MODELS}

Cox's (1972) proportional hazard model is widely used to examine various problems of survival analysis. However, we cannot use the proportional hazard model when heterogeneity of the baseline hazard function exists. For the LOS, variances are often heterogeneous even after controlling for characteristics of diseases, treatments, and patients. Therefore, it is improper to use the proportional hazard model for such a data set. We present a new model that is a modification of the model of Nawata et al. (2006). Let $i$ be the sample period and $n_{i}$ be the number of patients in the period $i$. (Since we consider two periods before and after the introduction of the DPC system, the value of $i$ is either 1 or 2.) Suppose that the revenue and cost of the hospital are $b_{i j}$ and $c_{i j}$, respectively. The revenue includes not only direct monetary payments but also improvements in its asset value owing to high-quality medical services, and the cost also includes an opportunity cost arising from the loss of revenue that the hospital suffers because of the unavailability of beds for new patients. Let

$$
g\left(t, x_{i j}, v_{i j}\right)=\frac{\partial c_{i j}}{\partial t}-\frac{\partial b_{i j}}{\partial t},
$$


where $t$ is the LOS, $x_{i j}$ is a vector of explanatory variables affecting the hospital's revenue and cost, and $v_{i j}$ is an unobserved error term. Here, $g(t)$ is assumed to be an increasing function of $t$. Following Nawata et al. (2006), we assume that

$$
z_{i j}(t) \equiv g\left(t, x_{i j}, v_{i j}\right)=t^{\alpha}-\left(x_{i j}{ }^{\prime} \beta+v_{i j}\right), \quad \alpha \geq 0 .
$$

When patients who stay at hospitals for long periods exit, $\alpha$ becomes less than one. The variances are quite different depending on the sample period. Hence we do not assume homoskedasticity of variances, and $v_{i j}$ is assumed to follow the normal distribution with mean 0 and variance $\sigma_{i}^{2}$. ( If we put $\left(t^{\alpha}-1\right) / \alpha-\left(x_{i j}{ }^{\prime} \beta^{*}+v_{i j}^{*}\right)$ and $v_{i j}^{*}=v_{i j} / \alpha$, we get an identical model. Thus, we can consider the model to be a version of the Box-Cox (1964) transformation model widely used in various fields.) We insert a minus sign and make $-\left(x_{i j}{ }^{\prime} \beta+v_{i j}\right)$ in Equation (2) so that the LOS becomes longer as the value of $x_{i j}{ }^{\prime} \beta$ becomes larger.

The LOS is a discrete variable taking positive integers. The condition for the $j$ th patient in the sample period $i$ being discharged from the hospital on $t_{i j}$ is given by

$$
z_{i j}\left(t_{i j}\right) \geq 0 \text { if } t_{i j}=1 \text {, and } z_{i j}\left(t_{i j}-1\right)<0, z_{i j}\left(t_{i j}\right) \geq 0 \text { if } t_{i j}>1 \text {. }
$$

Further, to remove the influence of a small number of patients who remained in the hospital over a long period of time, we limit the maximum number of days that patients could stay at the hospital to $T$. For patients staying more than $T$ days, we only use the information that their stay in the hospital exceeded $T$ days.

The probability of the patient being discharged from the hospital on $t_{i j}$ is given by

$$
P_{i j}=P\left[t_{i j}^{\alpha}-x_{i j}{ }^{\prime} \beta \geq v_{i j}\right] \text { if } t_{i j}=1 \text {, and } P\left[\left(t_{i j}-1\right)^{\alpha}-x_{i j}{ }^{\prime} \beta<v_{i j} \leq t_{i j}{ }^{\alpha}-x_{i j}{ }^{\prime} \beta\right] \text { if } 1<t_{i j} \leq T \text {. }
$$

The probability of the patient staying at the hospital for more than $T$ days is given by $P\left[T^{\alpha}-\left(x_{i j}{ }^{\prime} \beta+v_{i j}\right)\right]$. Let $\Phi$ be the distribution function of the standard normal distribution. We get the likelihood function given by

$$
\begin{aligned}
& L\left(\alpha, \beta, \sigma_{i}\right)=\prod_{t_{i j}=1}\left[\Phi\left\{\left(t_{i j}{ }^{\alpha}-x_{i j}{ }^{\prime} \beta\right) / \sigma_{i}\right\}\right] \\
& \times \prod_{1<t_{i j} \leq T}\left[\Phi\left\{\left(t_{i j}^{\alpha}-x_{i j}{ }^{\prime} \beta\right) / \sigma_{i}\right\}-\Phi\left[\left\{\left(t_{i j}-1\right)^{\alpha}-x_{i j}{ }^{\prime} \beta\right\} / \sigma_{i}\right]\right] \times \prod_{t_{i j}>T}\left[1-\Phi\left\{\left(T^{\alpha}-x_{i j}{ }^{\prime} \beta\right) / \sigma_{i}\right\}\right] .
\end{aligned}
$$

However, the function is a complicated form of parameters, and estimation of the model is not easy. In this paper, we propose a new model that can easily make the estimation using a standard statistical package program. The method is based on an approximation of the likelihood function. Considering the first-order Taylor expansion, we get

$$
\Phi\left\{\left(t_{i j}^{\alpha}-x_{i j}{ }^{\prime} \beta\right) / \sigma_{i}\right\}-\Phi\left[\left\{\left(t_{i j}-1\right)^{\alpha}-x_{i j}{ }^{\prime} \beta\right\} / \sigma_{i}\right] \approx \frac{1}{\sigma_{i}} \phi\left[\left\{\left(t_{i j}-\frac{1}{2}\right)^{\alpha}-x_{i j}{ }^{\prime} \beta\right\} / \sigma_{i}\right] \alpha\left(t_{i j}-\frac{1}{2}\right)^{\alpha-1} .
$$

To make the approximation more accurate, we evaluate the derivative at $t_{i j}-1 / 2$. When the value of $\Phi\left(-x_{i j}{ }^{\prime} \beta / \sigma_{i}\right)$ is small, Equation (6) can be used for $t_{i j}=1$. The log of the likelihood function is approximated by

$$
\begin{aligned}
& \log L^{*}\left(\alpha, \beta, \sigma_{i}\right)=\sum_{t_{i j} \leq T}\left[\log \phi\left[\left\{\left(t_{i j}-\frac{1}{2}\right)^{\alpha}-x_{i j}{ }^{\prime} \beta\right\} / \sigma_{i}\right]-\log \sigma_{i}\right] \\
& +\sum_{t_{i j} \leq T}^{n}\left\{\log \alpha+(\alpha-1) \log \left(t_{i j}-\frac{1}{2}\right)\right\}+\sum_{t_{i j}>T} \log \left[1-\Phi\left\{\left(T^{\alpha}-x_{i j}{ }^{\prime} \beta\right) / \sigma_{i}\right\}\right] .
\end{aligned}
$$


The second term of Equation (7) does not depend on $\beta$. When $\alpha$ is given, the estimator $\hat{\beta}_{\alpha}^{*}$, which maximizes $\log L^{*}$, is obtained by maximizing

$$
\log L_{\alpha}\left(\beta, \sigma_{i}\right)=\sum_{\tau_{i j}<c}\left[\log \phi\left[\left\{\left(\tau_{i j}-x_{i j}{ }^{\prime} \beta\right\} / \sigma_{i}\right]-\log \sigma_{i}\right]+\sum_{\tau_{i j} \geq c} \log \left[1-\Phi\left\{\left(c-x_{i j}{ }^{\prime} \beta\right) / \sigma_{i}\right\}\right],\right.
$$

where $\tau_{i j}=\left(t_{i j}-\frac{1}{2}\right)^{\alpha}$ and $c=T^{\alpha}$. Suppose that the first element of $x_{i j}$ is 1 . Let $\tau_{i j}^{*}=c-\tau_{i j}$, and $\beta^{-}$be a vector of parameters given by

$$
\beta_{1}^{-}=c-\beta_{1} \text {, and } \beta_{m}^{-}=-\beta_{m} \text { if } m \geq 2
$$

where $\beta_{m}$ and $\beta_{m}^{-}$are the $m$ th elements of $\beta$ and $\beta^{-}$, respectively. In this case the model becomes a standard tobit model (censored regression model) given by

$$
\tau_{i j}^{* *}=-x_{i j}{ }^{\prime} \beta+c+\varepsilon_{i j} \equiv x_{i j}{ }^{\prime} \beta^{-}+\varepsilon_{i j}, \tau_{i j}^{*}=\max \left\{\tau_{i j}^{* *}, 0\right\}, \text { and } \varepsilon_{i j} \sim N\left(0, \sigma_{i}^{2}\right) .
$$

The estimator $\hat{\beta}_{\alpha}$, which has the same asymptotic distribution as $\hat{\beta}_{\alpha}^{*}$, can be easily obtained by the following steps using a standard statistical package program:

i) Estimate Equation (10) by the tobit maximum likelihood method, and calculate $\hat{\sigma}_{i}^{2}$, which is the estimator of $\sigma_{i}^{2}$, for each value of $i$.

ii) Adjust the heteroskedasticity by dividing variables by $\hat{\sigma}_{i}$.

iii) Estimate $\beta^{-}$using the adjusted data set, and calculate $\hat{\beta}_{\alpha}^{*}$ from Equation (9). Note that the standard tobit maximum likelihood estimator is not consistent under heteroskedasticity, so it is necessary to estimate $\hat{\sigma}_{i}^{2}$ and adjust the heteroskedasticity. Next, we can obtain estimators $\hat{\alpha}$ and $\hat{\beta}$ by maximizing $\log L^{*}\left(\alpha, \hat{\beta}_{\alpha}\right)$ with respect to $\alpha$. Here, considering the possibility that $\log L^{*}\left(\alpha, \hat{\beta}_{\alpha}\right)$ has plural local maxima, we employ the scanning method of Nawata (1994) and Nawata and Nagse (1996) for $0<\alpha \leq 1$.

\section{ESTIMATION RESULTS}

We consider two different periods. The periods $i=1$ and $i=2$ mean the first period and second periods, respectively. We choose the following variables as explanatory variables. The Female Dummy (0: male, 1: female) was used for sex. The proportion of females was $62.3 \%$ and $63.6 \%$ for the first and second periods, respectively. Concerning ages of patients, we used the following age dummies: Under 40 (1: under 40, 0: otherwise), Age 40 (1: 40-49, 0: otherwise), Age 50 (1: 50-59, 0: otherwise), Age 60 (1: 60-69, 0: otherwise), Age 70 (1: 70-79, 0 otherwise), Age 80 (1: 80-89, 0 otherwise), and Age 90 (1: 90 or over, 0: otherwise). The average age of the patients was (standard deviations are in parentheses) 73.7 (9.7) and 75.4 (8.8) for the first and second periods, respectively. The average age in the second period was 1.7 years higher than that in the first period. To represent the place to which patients went after hospitalization, we used the Not_Home Dummy (0: going back to home, 1: otherwise). In this study, 37 and 2 patients did not go back home for the first and second periods, respectively. Four Hpk dummies (1: Hpk, 0: otherwise, $k=2,3,4,5)$ were used to represent the influences of hospitals. The base of these variables is Hp1, where the ALOS was the shortest in the first period. For the influence of sample periods, the Second Period Dummy (0: first period, 1: second period) was used. Since the influences of the DPC system might be different among hospitals, the product of the hospital dummies and Second Period Dummy ( $\mathrm{Hp} k$ Dummies $\times$ Second Period Dummy) were also included as explanatory variables. Therefore, $x_{i j}{ }^{\prime} \beta$ of Equation (2) becomes

$$
\begin{aligned}
x_{i j}{ }^{\prime} \beta & =\beta_{1}+\beta_{2} \text { Female Dummy }+\beta_{3} \text { Under } 40 \text { Dummy }+\beta_{4} \text { Age } 40 \text { Dummy }+\beta_{5} \text { Age } 50 \text { Dummy } \\
& +\beta_{6} \text { Age } 60 \text { Dummy }+\beta_{7} \text { Age } 80 \text { Dummy }+\beta_{8} \text { Age } 90 \text { Dummy }+\beta_{9} \text { Not_Home Dummy } \\
& +\beta_{10} \text { Second Period Dummy }+\sum_{k} \beta_{k} \text { Hp } k \text { Dummy }
\end{aligned}
$$




$$
+\sum_{k} \beta_{k}(\mathrm{Hp} k \text { Dummy } \times \text { Second Period Dummy }) .
$$

To remove the influence of the small number of patients who stayed at the hospital over a long period of time, we select $T=12$. A total of 57 patients $-2.2 \%$ of all patients - stayed at the hospital more than 12 days.

Table 1 presents the estimates for $\alpha$ and $\beta$. The estimate for $\alpha$ is 0.5322 and significantly smaller than 1.0, which implies that some patients remained at the hospital for a long period of time. The estimate for the Female Dummy is positive and significant at the $5 \%$ level. This implies that the female patient stays at the hospital longer than the male patient. Concerning ages, the estimate for the Age 50 Dummy is negative and significant at the $1 \%$ level and that for the Age 90 dummy is positive and significant at the $5 \%$ level. The estimate for the Under 40 Dummy is negative, and the estimates for the Age 40 and Age 80 dummies are positive but not significant at the $5 \%$ level. The estimate for the Not_Home Dummy is positive and significant at the $1 \%$ level, and the LOS becomes longer if the patient does not go back home after hospitalization.

All estimates of the Hospital dummies are positive and significant at the $1 \%$ level. The values (since the Hp1 is the base of the Hospital dummies, the value of $\mathrm{Hp} 1$ is set to 0 ) and orders of these estimates are almost proportional to the ALOSs of hospitals (the correlation coefficient is 0.908 ). This implies that the LOS of each hospital does not change much, even if the influence of patient characteristics is eliminated in the first period. In other words, despite the exclusion of the effects of patient characteristics, large differences remain among hospitals. The estimate of the Second Period Dummy is positive and significant. This implies that the LOS does not decrease and rather increases in the second period for Hp1. On the other hand, the estimates of the (Hp $k$ Dummy $\times$ Second Period Dummy) become negative and significant at the $1 \%$ level for Hp2-Hp5. The changes in the LOS for these hospitals in the second period are given by the sum of estimates of the Second Period Dummy and (Hp $k$ Dummy $\times$ Second Period Dummy). These values are $-0.414 \sim-0.594$. Their $t$-values are $-3.568 \sim-12.112$, and they are significant at the 1\% level. This implies that the LOSs for these hospitals became shorter in the second period. Moreover, the reduction was larger as the ALOS in the first period was longer. This result is consistent with the fact that per diem payment becomes less as the LOS becomes longer. It suggests that the DPC system gave strong incentives to reduce the LOS for a hospital where the ALOS was long, but weak (or no) incentives for a hospital where the ALOS was already short (we refer to the former and latter types of hospital as the short and long ALOS hospitals, respectively).

The estimates of $\sigma_{i}$ decrease from $\hat{\sigma}_{1}=0.422$ in the first period to $\hat{\sigma}_{2}=0.227$ in the second period. The $t$ value for the null hypothesis of the homogeneity of the variance is quite large (22.30). This hypothesis is rejected at the (any reasonable) significant level. It is admitted that the variance decreased in the second period, and feasibility of the proposed model is strongly suggested.

\section{CONCLUSION}

In this paper, we proposed a model for analyzing the LOS at hospitals. This model is an alternative to the conventional models such as Cox's proportional hazard model and can be used to analyze various problems of survival analysis. The model is a tobit-type model, and its estimation can be easily done using a standard statistical package program. Using the proposed model, we analyzed the effects of the DPC system on the LOS for cataract operations in Japan using data collected from 5 general hospitals. The estimates of the Female, Age 50, Age 90, Not Home dummies are significant and affect the LOS. We found large differences in the changes of ALOSs among hospitals. For the short ALOS hospitals, the ALOSs did not decrease. On the other hand, for the long ALOS hospitals, the ALOS decreased significantly. The results of empirical study imply that the DPC system gave strong incentives to reduce the LOS for the long ALOS hospitals, but it gave weak (or no) incentives for the short ALOS hospitals where the ALOSs were already short.

In this study, we only surveyed five hospitals. The sample selection bias of hospitals may be a problem. However, since the number of hospitals is small, we cannot analyze the effect of sample selection bias. Although obtaining data from before the introduction of the DPC system is very difficult, it is necessary to collect data for more hospitals to evaluate the DPC system more precisely. We also need to perform the same analysis for other diseases. These are subjects for future studies. 
Nawata et al., Evaluation of the DPC-based inclusive payment system in Japan for cataract operations

\section{REFERENCES}

Box, G. E. P., and Cox, D. R. (1964) "An analysis of transformation," Journal of the Royal Statistical Society $B, 26,211-252$.

Cox, D. R. (1972) “Regression models and life tables,” Journal of Royal Statistical Society B, 34: 187-220.

Fedorowicz, Z., Lawrence, D. J., and Guttie, P. (2006) “A Cochrane systematic review finds no significant difference in outcome or risk of postoperative complications between day care and inpatient cataract surgery," Saudi Medical Journal, 27, 1296-1301.

Ministry of Health, Labour and Welfare, (2008) Patient Survey 2006.

Nawata, K. (1994) "Estimation of sample selection bias models by the maximum likelihood estimator and Heckman's two-step estimator," Economic Letters, 45, 33-40.

Nawata, K., and Nagase, N. (1996) "Estimation of sample selection biases models," Econometric Reviews, 15, 387-400.

Nawata, K., Niita, A., Watanabe, S., and Kawabuchi, K. (2006) "An analysis of the length of stay and the effectiveness of treatment for hip fractured patients in Japan: evaluation of the revision of the 2002 medical service fee schedule," Journal of Health Economics, 25, 722-739.

Okamura, S., Kobayashi, R., and Sakai, T. (2005) “Case-mix payment in Japanese Medicare,” Health Policy, 74, 282-285.

Yasunaga, H., Ide, Y. H. Imamura, T., et al. (2005) "Impact of the Japanese diagnosis procedure combination-based system on the cardiovascular medicine-related costs," International Heart Journal, 46, 855-866.

Table 1. Results of estimation

\begin{tabular}{|c|c|c|c|c|c|}
\hline Variable & Estimate & Standard error & Variable & Estimate & Standard error \\
\hline Constant & 1.8897 & 0.01731 & \multicolumn{3}{|c|}{ Hospital Dummies } \\
\hline Female Dummy & 0.034 & 0.01524 & Hp4 & 0.4744 & 0.06828 \\
\hline \multicolumn{3}{|c|}{ Age Dummies } & Hp5 & 1.0646 & 0.04309 \\
\hline Under 40 & -0.1658 & 0.09616 & Second Period Dummy & 0.0264 & 0.01284 \\
\hline Age 40 & 0.1 & 0.0909 & \multicolumn{3}{|c|}{ Hp $k$ Dummies $\times$ Second Period Dummy } \\
\hline Age 50 & -0.0929 & 0.03437 & Hp2 & -0.4405 & 0.11738 \\
\hline Age 60 & -0.0169 & 0.0201 & Hp3 & -0.5538 & 0.04705 \\
\hline Age 80 & 0.029 & 0.0177 & Hp4 & -0.5461 & 0.07828 \\
\hline Age 90 & 0.124 & 0.05297 & Hp5 & -0.6209 & 0.07379 \\
\hline Not_Home Dummy & 0.4513 & 0.06271 & $\alpha$ & 0.5322 & 0.01441 \\
\hline \multicolumn{3}{|c|}{ Hospital Dummies } & $\sigma_{1}$ & 0.4346 & 0.00799 \\
\hline Hp2 & 0.8382 & 0.04773 & $\sigma_{2}$ & 0.2256 & 0.00511 \\
\hline Hp3 & 0.6251 & 0.0235 & $\log L^{*}$ & \multicolumn{2}{|c|}{-4541.549} \\
\hline
\end{tabular}

